Chirurg 2012 $\cdot 83: 1085$

DOI 10.1007/s00104-012-2401-z

Online publiziert: 11. November 2012

c) Springer-Verlag Berlin Heidelberg 2012
J. Reibetanz · C.-T. Germer

Klinik für Allgemein-, Viszeral-, Gefäß- und Kinderchirurgie, Universitätsklinikum Würzburg

\section{Outcome nach laparoskopischer vs. offener abdominoperinealer Rektumextirpation}

\section{Ergebnisse einer Metaanalyse}

\section{Originalbeitrag}

Racheva G, Elmusharaf H (2012) A systematic review and meta-analysis of randomized and non-randomized studies comparing laparoscopic and open abdominoperineal resection for rectal cancer. Colorectal Dis. DOI 10.1111/codi.12007

Die sphinktererhaltende Resektion stellt, wo immer möglich, den Standard in der Rektumkarzinomchirurgie dar. Die breite Anwendung der offenen und laparoskopischen anterioren Rektumresektion (AR) in Klinik und Literatur lässt jedoch - unter den Kriterien der evidenzbasierten Medizin - die Datenbasis für die abdominoperineale Rektumextirpation (APR) eher dürftig erscheinen, obwohl letztere für immerhin 15\% der Rektumkarzinome die onkologisch bessere Alternative darstellt.

\section{Fragestellung und Methode}

Ziel dieser systematischen Literaturrecherche war der Vergleich der Kurzzeitund Langzeitergebnisse zwischen laparoskopischer und offener APR. Primärer Endpunkt war die Rate an Lokal- bzw. systemischen Rezidiven beider Techniken, sekundäre Endpunkte beinhalteten das Gesamtüberleben, die onkologische Radikalität und das perioperative Outcome. Eingeschlossen wurden sowohl randomisierte als auch nichtrandomisierte Studien ohne Einschränkung hinsichtlich Sprache oder Publikationszeitraum.

\section{Ergebnisse}

Von insgesamt 67 identifizierten Studien wurden 3 randomisiert-kontrollier- te und 5 nichtrandomisierte Studien in die Metaanalyse eingeschlossen. Diese beinhalteten 206 Patienten nach offener und 248 Patienten nach laparoskopischer APR mit einem Follow-up von 49 (Range: 22-88) bzw. 44 (12-87) Monaten. Die Ergebnisse zeigen eine niedrigere Rate an Lokal- (Odds Ratio [OR]: 2,73; 95\%-Konfidenzintervall [KI]: 1,14-6,58; $\mathrm{p}=0,025)$ und systemischen Rezidiven (OR: 1,99; 95\%-KI: 1,06-3,74; $\mathrm{p}=0,032)$ nach laparoskopischer APR, wobei dieser Unterschied verschwand, wenn in die Analyse nur die randomisierten Studien eingeschlossen wurden. Beide Techniken waren vergleichbar hinsichtlich des Gesamtüberlebens ( $\mathrm{p}=0,17)$, der Anzahl entfernter Lymphknoten ( $\mathrm{p}=0,73)$, der Präparatelänge $(\mathrm{p}=0,28)$ und der Rate an positiven zirkumferenziellen Resektionsrändern $(\mathrm{p}=0,89)$. Früh-postoperative Komplikationen traten signifikant seltener nach laparoskopischer APR auf ( $\mathrm{p}=0,001)$, wohingegen sich die beiden Techniken im Hinblick auf intraoperative Komplikationen, perineale Wundheilungsstörungen und Anzahl von Reoperationen nicht unterschieden. Die Dauer des postoperativen Ileus und die Zeit bis zur kompletten Mobilisation waren erwartungsgemäß beim laparoskopischen Zugang kürzer.

\section{Diskussion und Fazit des Reviewers}

Es ist bekannt, dass die onkologischen Ergebnisse der APR im Vergleich zur AR schlechter ausfallen, was v. a. durch eine Selektion zuungunsten der APR bedingt ist (lokal-fortgeschrittenere und tiefer sitzende Tumoren), jedoch auch operationstechnische Gründe hat (Taillierung des Mesorektums im Bereich des anorektalen Übergangs mit höherem R1-Risiko bei der APR). Die vorliegende Metaanalyse konnte nun zeigen, dass es zudem innerhalb der APR in Abhängigkeit des operativen Zugangs weitere Unterschiede hinsichtlich gewisser perioperativer Parameter, aber auch in Bezug auf das Rezidivrisiko zu geben scheint, und das jeweils mit Bevorzugung der minimal-invasiven Technik. Ersteres mag nicht verwundern und ist aus randomisierten Studien zum Vergleich der offenen und laparoskopischen Kolorektalchirurgie bekannt. Im Gegensatz dazu erstaunen die geringeren lokalen und systemischen Rezidivraten nach laparoskopischer APR etwas, zumal der "onkologische" Part bei der APR von perineal aus erfolgt und der abdominelle Teil der Operation, ob nun laparoskopisch oder offen durchgeführt, wenig zur (Lokal-)Rezidivvermeidung beiträgt. Dies mag erklären, warum sich (auch) in dieser Metaanalyse zum Vergleich zwischen offener und minimal-invasiver kolorektaler Karzinomchirurgie das Gesamtüberleben nicht unterscheidet.

\section{Korrespondenzadresse}

\section{Prof. Dr. C.-T. Germer}

Klinik für Allgemein-, Viszeral-, Gefäß- und Kinderchirurgie, Universitätsklinikum Würzburg, Oberdürrbacher Str. 6, 97080 Würzburg germer_c@klinik.uni-wuerzburg.de

Interessenkonflikt. Der korrespondierende Autor gibt für sich und seinen Koautor an, dass kein Interessenkonflikt besteht. 